\title{
The distribution of human papillomavirus in tissues from patients with head and neck squamous cell carcinoma
}

\author{
WEI WEI ${ }^{1^{*}}$, QI SHI $^{2^{*}}$, FEI GUO $^{2 *}$, BAO-YUN ZHANG $^{2}, \mathrm{CAO} \mathrm{CHEN}^{2}$, \\ NAI-SONG ZHANG ${ }^{1}$ and XIAO-PING DONG ${ }^{2,3}$
}

\begin{abstract}
${ }^{1}$ Key Laboratory of Carcinogenesis and Translational Research (Ministry of Education), Head and Neck Surgery Department Peking University Cancer Hospital and Institute, Beijing 100142; ${ }^{2}$ State Key Laboratory for Infectious Disease Prevention and Control, National Institute for Viral Disease Control and Prevention, Chinese Center for Disease Control and Prevention,

Beijing 102206; ${ }^{3}$ Chinese Academy of Sciences Key Laboratory of Pathogenic Microbiology and Immunology, Institute of Microbiology, Chinese Academy of Sciences, Beijing 100101, P.R. China
\end{abstract}

Received April 11, 2012; Accepted June 14, 2012

DOI: $10.3892 /$ or.2012.1990

\begin{abstract}
Several types of HPVs have been shown to be associated with the development of malignant cancers in various head and neck tumors. More information on the HPV prevalence in patients with head and neck squamous cell carcinomas (SCCs) need to be obtained. In this study, formalin-fixed and paraffin-embedded tissues of 93 pathologically diagnosed head and neck SCC patients were collected from Peking University Cancer Hospital. HPV DNA sequences in tumor tissues were screened by a commercial Luminex technique for HPVs and HPV-specific PCR assays. Presence of HPV16/18 oncoprotein in tumor tissues was assessed by immunohistochemistry (IHC) with HPV16/18 E6-specific antibodies. Of the 93 patients, $16(17.2 \%)$ cases were found to be HPV DNA-positive, including 7 HPV18-positive, 8 HPV16-positive and 1 HPV52-positive. IHC assays demonstrated that $31.2 \%$ (29/93) tested sections showed positive signals in the tumor cells. The total positive rate of HPV genome and its encoding products in the tested samples was $44.1 \%$ (41/93). Further analyses revealed that HPV infections in head and neck SCCs were significantly related with the tumor anatomic sites, showing decreasing tendency
\end{abstract}

Correspondence to: Dr Nai-Song Zhang, Key Laboratory of Carcinogenesis and Translational Research (Ministry of Education), Head and Neck Surgery Department, Peking University Cancer Hospital and Institute, Beijing 100142, P.R. China

E-mail: Zhangns66@yahoo.com.cn

Dr Xiao-Ping Dong, State Key Laboratory for Infectious Disease Prevention and Control, National Institute for Viral Disease Control and Prevention, Chinese Center for Disease Control and Prevention, Chang-Bai Rd 155, Beijing 102206, P.R. China

E-mail: dongxp238@sina.com

*Contributed equally

Key words: head and neck squamous cell carcinoma, human papillomavirus, DNA detection, Luminex, oncoprotein from outside (lip cancer) to inside (laryngeal cancer), but had no correlation with pathological, clinical grades and age of the patients. In all, HPV infections are commonly identified in the tumor tissues of patients with head and neck SCCs, in which HPV16 and 18 are the most prevalent HPV genotypes. Direct detection of high-risk HPV oncoprotein by IHC may be a good tool for classifying a tumor as truly HPV-associated.

\section{Introduction}

Head and neck cancers are a group of relatively common malignant diseases worldwide, which occupies $\sim 5 \%$ human malignant carcinomas. In China, the morbidities of lip, oropharynx, hypopharynx and larynx carcinoma were evaluated as $0.04-0.14$, $1-2,0.15-0.8$ and 3-5/100000, respectively. Roughly $85-90 \%$ of oral and oropharyngeal cancers are histologically squamous cell carcinomas (SCC). SCC most commonly affects men from 50 to 70 years of age and is associated with alcohol and tobacco consumption. The distributions of head and neck cancers in China show clearly geography difference, that oral and larynx cancers are commonly identified in the northern part of China and nasopharyngeal cancer, thyroid cancer, malignant lymphoma and skin cancer are predominant in the southern part of China (1).

Human papillomavirus (HPV) is a small, epitheliotropic, double-stranded DNA virus, whose whole genome is $\sim 8 \mathrm{~kb}$ long. Up to now, 150 HPV genetic subtypes have been described. HPVs possess great importance in human carcinogenesis since the 1970s when it was discovered to be the causative agent of cervical cancer. Based on the propensity to immortalize human keratinocyte cell lines and override cell cycle control mechanisms, HPVs are classified into low, mediate and high-risk categories. Besides the direct evidence that infections of highrisk HPVs are etiologically associated with the carcinogenesis of cervical cancer, more and more data have supported that HPV infection is also closely related with other human SCCs, especially with head and neck SCCs, among them HPV16 is associated with more than $90 \%$ of HPV-related head and neck cancers (2). Other types less frequently detected in oropharyngeal cancers include HPV6, 18, 31, 33, 35, 45, 52 and 58 (3). 
Recently, an Australian group has reported an increasing incidence of potentially HPV-associated oropharyngeal cancers in Australia between 1982 and 2005 (4).

During the past few decades, HPV DNA has been detected in $25 \%$ of head and neck SCCs overall. More importantly, $45-100 \%$ oral SCC cases were reported to be HPV-positive. The variations of HPV distributions in SCCs may relate with different factors, such as the location of cancers, the type of specimens available, the techniques used for testing and the time period and country or region the SCC specimens obtained (5). The prevalence of HPVs in Chinese patients with head and neck SCCs has been also described. Using various techniques, including PCR, hybridization in situ and immunohistochemistry (IHC), HPV-positive rates vary from 33.3 to $72.6 \%$ among various SCCs, such lip, oral cavity, orophyrynx, hypophrynx and larynx cancers (6). In addition to the large variations of HPV-positive rates from independent groups, repeatedly identified HPV DNA sequences and their encoding proteins in the tissues of Chinese head and neck SCCs propose an obvious tendency that HPV infection plays also essential role in the pathogenesis of head and neck SCCs in China.

To get more information on the HPV prevalence in Chinese head and neck SCCs, 93 head and neck SCCs were screened with HPV specific Luminex technique, PCR and IHC assays, 44.1\% (41/93) tested samples showed HPV-positive. Further analyses revealed that the HPV-positive rates had no correlation with the pathological and clinical grades of cancers, but showed significant relation with the anatomic sites of tumors. Additionally, the distribution of HPVs in various age groups of the patients with head and neck SCCs was similar.

\section{Patients and methods}

Patients and specimens. The specimens from the 93 patients with malignant squamous tumors, including 64 laryngocarcinoma, 5 oropharyngeal carcinoma, 15 hypopharynx carcinoma and 9 lip carcinoma were included in this study. All cases were hospitalized in the Head and Neck Surgery Department, Peking University Cancer Hospital and Institute, from 2006 to 2011. Among them, 84 were male and 9 were female. The ages of the patients ranged from 27 to 84 years, with the median of 57 years, 87.1\% (81/93) and $57.0 \%$ $(53 / 93)$ of the patients had histories of tobacco or alcohol use. The professions of the patients varied largely, and the permanent residences of the patients were distributed widely in mainland of China. All samples were surgically removed and conventionally fixed in $10 \%$ formalin and paraffin embedded. Pathological assays verified that all cancers were squamous cancers. The pathological grades of the cancers were assessed by clinical pathologists in the Peking University Cancer Hospital and Institute. The clinical grades of patients with tumors were finally determined by surgeons in the Peking University Cancer Hospital and Institute.

DNA extraction. Total DNAs from the tumor tissues were extracted from the paraffin-embedded tissue blocks with a commercial genomic DNA extraction FFPE kit (Qiagen). Briefly, 3-4 formalin-fixed, paraffin-embedded $10 \mu \mathrm{m}$ sections were soaked in xylene vortex vigorously for at least $1 \mathrm{~h}$. Then pellet was acquired and purified under the kit protocol. Quality of the extracted DNAs was assessed with a settled PCR protocol with a pair of actin-specific primers, which produced a 240-bp long fragment.

Luminex technique for HPVs DNA. The presences of HPVs specific DNA sequences in the extracted DNAs from the tested tumors were screened with a Tellgenplex ${ }^{\mathrm{TM}}$ HPV DNA Test kit (Tellgen, China) using Luminex technique, which allowed to detect 26 genotypes of HPVs including 19 high-risk HPV types (HPV16, 18, 26, 31, 33, 35, 39, 45, 51, 52, 53, 55, 56, 58, 59, 66, 68, 82 and 83) and 7 low-risk HPV types (HPV6, 11, 40, 42, 44, 61 and 73). The experiments were performed in Bio-Plex 200 system according to the manufacturer's instructions. Briefly, $2 \mu \mathrm{g}$ of the extracted DNA was proceeded the PCR protocol as following: $95^{\circ} \mathrm{C} 30 \mathrm{sec}, 58^{\circ} \mathrm{C} 30 \mathrm{sec}$ and $72^{\circ} \mathrm{C}$ $30 \mathrm{sec}$ for the first 5 circles, and $95^{\circ} \mathrm{C} 30 \mathrm{sec}, 55^{\circ} \mathrm{C} 30 \mathrm{sec}$ and $72^{\circ} \mathrm{C} 30 \mathrm{sec}$ for another 35 circles. The PCR products were subjected into a fast hybridization and the data were analyzed with the software supplied by the manufacturer.

PCR protocols for HPV16 and HPV18. Two-rounded PCR techniques for HPV16 and HPV18 were individually established. The primers were P16-1 (5'-GCAAGCAACAG TTACTGCGA-3') and P16-2 (5'-CAACAAGACATACATCG ACC-3') for HPV16 E6 sequence and P18-1 (5'-CACTTCACT GCAAGACATAGA-3') and P18-2 (5'-GTTGTGAAATCGT CGTTTTTCA-3') for HPV18 E6 sequence. Extracted DNAs $(1.0 \mu \mathrm{g})$ were mixed with $1 \mu \mathrm{M}$ of individual primers and 1.25 U Pfu DNA polymerase in a $25 \mu 1$ volume. PCR was performed with the following conditions: $94^{\circ} \mathrm{C} 1 \mathrm{~min}, 57^{\circ} \mathrm{C} 1$ min and $72^{\circ} \mathrm{C} 1 \mathrm{~min}$, in total 30 cycles, and another $10-\mathrm{min}$ extension. For second round PCR, $5 \mu \mathrm{l}$ of the first round PCR product was mixed again with $10 \mu \mathrm{M}$ of individual primers and 1.25 U Pfu DNA polymerase in a $25 \mu 1$ volume. The PCR condition was $94^{\circ} \mathrm{C} 1 \mathrm{~min}, 52^{\circ} \mathrm{C} 1 \mathrm{~min}$ and $72^{\circ} \mathrm{C} 1 \mathrm{~min}$, totally 40 cycles and another $10 \mathrm{~min}$ extension. The targeting fragments of HPV16 E6 and HPV18 E6 were 333 and 322 bp, respectively. All PCR assays were carefully carried out in the PCR laboratory with four separating rooms to avoid of DNA contamination. Actin-specific 240-bp long fragment was produced as control.

Direct sequencing. The PCR products were analyzed in $2 \%$ agarose gel and recovered from gel with QIAquick Gel Extraction kit (Qiagen). Direct sequencing was performed using the same PCR primers and the base sequences were read on ABI PRISMTM 3730XL DNA analyzer.

Immunohistochemistry (IHC). Paraffin sections $(5 \mu \mathrm{m})$ were deparaffinized in xylene for 5 min twice and gradually rehydrated routinely. Sections were quenched for endogenous peroxidases in $3 \% \mathrm{H}_{2} \mathrm{O}_{2}$ in methanol for $15 \mathrm{~min}$, pretreated with enzyme digestion antigen retrieval for $1 \mathrm{~min}$. After blocking in $1 \%$ normal goat serum, the sections were incubated overnight at $4^{\circ} \mathrm{C}$ with 1:500-diluted mAb for HPV16/18 E6 (Abcam). The sections were then incubated for $60 \mathrm{~min}$ with 1:1000-diluted HRP-conjugated goat anti-mouse secondary antibody (Vector Labs, USA), and visualized by incubation with 3,3-diaminobenzidine tetrahydrochloride (DAB). The slices were dehydrated and mounted in 


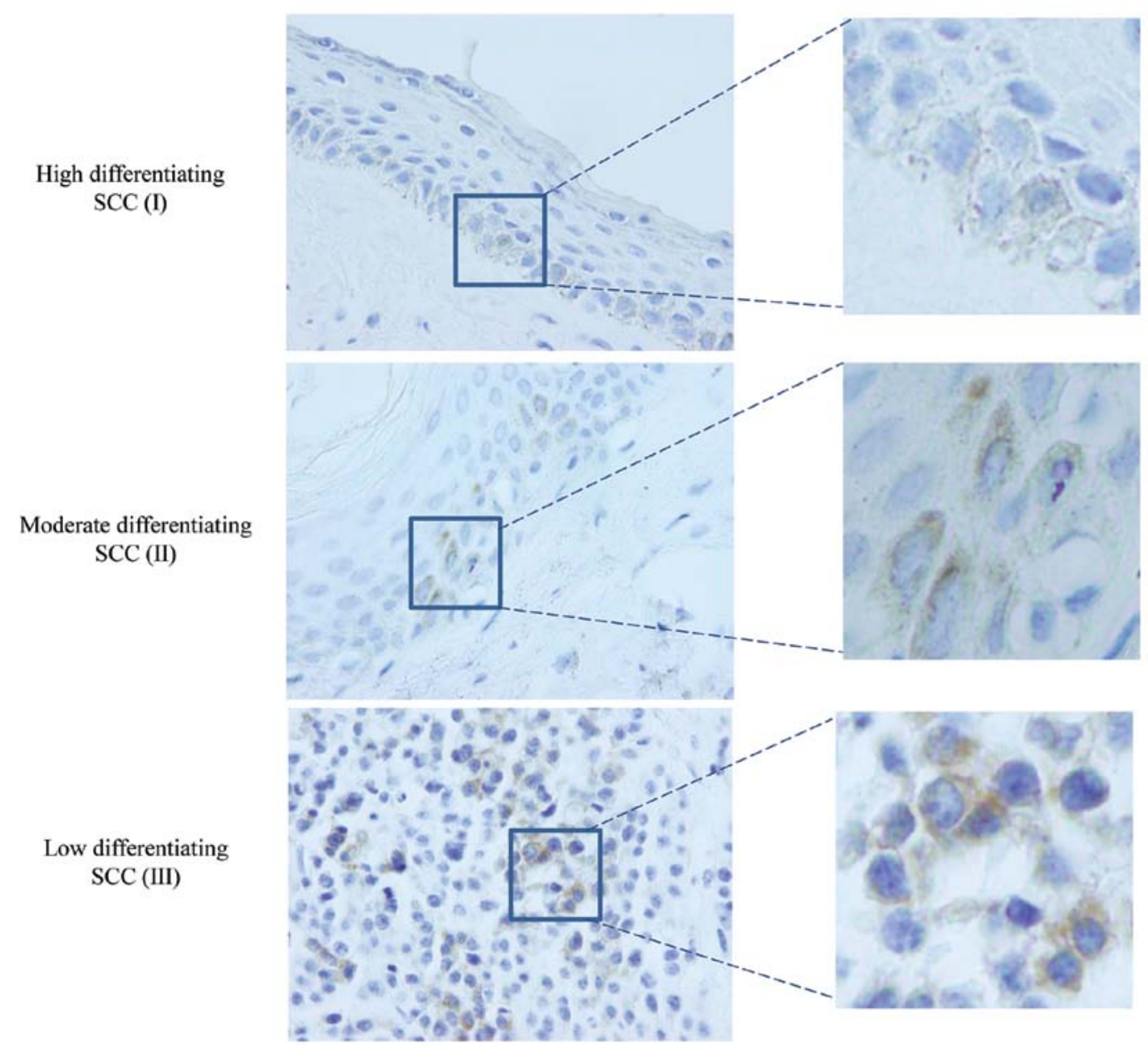

Figure 1. Immunohistochemistry assays of HPV16/18 oncoproteins in the tissues from various pathological grades of larynx SCC (x40). The magnified views are shown on the right of each picture. The pathological grades are indicated on the left.

Permount. Photomicrographs were taken with a DP70 digital camera mounted on BX5 microscope (Olympus Optical, Japan).

Statistical analyses. Statistical analyses were performed using SPSS (Statistical Package for the Social Sciences, Chicago, IL) 11.5 for windows. Prevalence of HPV infection in the head and neck squamous carcinomas of different sites, pathological grades, clinical grades and age groups were analyzed by the $\chi^{2}$ test. $\mathrm{P}<0.05$ was considered statistically significant.

Ethical statement. Written consent for further investigation and publication was obtained from the patients or the patients' relatives, respectively. Usage of the stored human samples in this study was approved by the Ethics Committees of Peking University Cancer Hospital and Institute and National Institute for Viral Disease Prevention and Control, China CDC.

\section{Results}

Identification of HPV-related sequences in the cancer tissues. The presence of HPV sequences in the fixed tumor tissues was firstly screened by a commercial Luminex technique for HPV genotyping. Out of 93 tumor samples, 7 cases showed positive HPV signals, in which 4 were HPV18-positive, 2 were HPV16- positive and 1 was HPV52-positive. To obtain more detailed evidence, the presence of HPVs sequences in tumor samples were further assayed with the established PCR protocols specific for HPV16, 18 and 52. Among the tested samples, 6 were HPV18-positive, 6 were HPV16-positive and 1 was HPV52positive. Sequencing assays of the positive PCR products were verified to be E6 sequences of the relative HPVs. Three out of 4 cases showing HPV18-positive in Luminex and the case showing HPV52-positive in Luminex were positive in the PCR assays, whereas 2 cases showing HPV16-positive in Luninex were PCR-negative. Collectively, 7 samples contained HPV18 specific sequences, 8 contained HPV16 sequences and one contained HPV52 sequences.

Identification of HPV-related proteins in the cancer tissues. To assess the HPV-related proteins in tumor tissues, the tissue sections of various cancers were immunohistochemically stained with mAb against HPV16/18 E6 oncoprotein. From 93 cancer cases, 29 samples (31.2\%) showed positive signals while none of the five polyps of larynx revealed positive signal. The brown-stained signals were present and restricted to cell nuclei and/or cytoplasm of the cancer cells, without detectable morphological difference among various pathological grades according to cell differentiation (Fig. 1). 
Table I. Prevalence of HPV infection in the head and neck squamous carcinomas of different sites.

\begin{tabular}{lcccr}
\hline & \multicolumn{4}{c}{ HPV-positive } \\
\cline { 2 - 4 } Site & HPV sequence (\%) & HPV protein $(\%)$ & HPV sequence + protein (\%) & Total (\%) \\
\hline Larynx & $7 / 64(10.9)$ & $18 / 64(28.1)$ & $2 / 64(3.1)$ & $23 / 64(35.9)$ \\
Hypopharynx & $6 / 15(40.0)$ & $2 / 15(13.3)$ & $1 / 15(6.7)$ & $7 / 15(46.7)$ \\
Oropharynx & $2 / 5(40)$ & $2 / 5(40)$ & $1 / 5(20)$ & $3 / 5(60.0)$ \\
Lip & $1 / 9(11.1)$ & $7 / 9(77.8)$ & $0 / 9(0.0)$ & $8 / 9(88.9)$ \\
Total & $16 / 93(17.2)$ & $29 / 93(31.2)$ & $4 / 93(4.3)$ & $41 / 93(44.1)^{\mathrm{a}}$ \\
\hline
\end{tabular}

${ }^{\mathrm{a}} \mathrm{P}=0.022$.

Table II. Prevalence of HPV infection in the squamous head and neck carcinomas according to their pathological grades.

\begin{tabular}{lcccr}
\hline Cell differentiation & High (I) $(\%)$ & Moderate (II) $(\%)$ & Low (III) $(\%)$ & P-value \\
\hline Larynx & $6 / 19(31.6)$ & $14 / 35(40.0)$ & $3 / 10(33.3)$ & 0.755 \\
Hypopharynx & $2 / 4(50.0)$ & $5 / 7(71.4)$ & $0 / 4(0.0)$ & 0.073 \\
Oropharynx & $2 / 2(100.0)$ & $0 / 2((0.0)$ & $1 / 1(100.0)$ & 0.082 \\
Lip & $4 / 4(100.0)$ & $3 / 4(75.0)$ & $1 / 1(100.0)$ & 0.495 \\
Total & $14 / 29(48.3)$ & $22 / 48(45.8)$ & $5 / 16(31.3)$ & 0.513 \\
\hline
\end{tabular}

Additionally, among 16 cancer samples containing HPV16 or 18 sequences, 4 were positive in IHC assay. This highlights a relatively high distribution of HPV associated proteins in the cancer tissues. However, the presence of HPV proteins and HPV gene sequences did not correlate with each other. The total positive rate of HPV genomes and its encoding products in the tested samples was $44.1 \%(41 / 93)$.

Correlations of HPV infections with tumor sites. Among the 93 tested cancers, 64 were laryngocarcinoma, 5 were oropharyngeal carcinoma, 15 were hypopharynx carcinoma and 9 were lip carcinoma. Table I summarized the results of HPV genomes and proteins in various cancers. The presences of HPV sequences and/or proteins in the cancer tissues seemed to differ largely based on the sites of the malignant tumors, $35.9 \%$ (23/64) laryngocarcinoma, 46.7\% (7/15) hypopharynx carcinoma, $60.0 \%$ (3/5) oropharyngeal carcinoma and $88.9 \%$ (8/9) lip carcinoma were HPV-positive either in the assays of viral nucleosides (Luminex and PCR) or in that of viral proteins (IHC). Statistical analysis revealed a significant difference $(\mathrm{P}=0.022)$ in the anatomic sites of tumors. It illustrates a clear declining manner of HPV infection in the head and neck tumors from outside (lip) to inside (larynx). Moreover, the positive rates of viral sequences and proteins were different among various tumors. The detection rates of HPV16/18 oncoprotein was significantly higher than that of HPVs DNA in lip cancers (77.8 vs $11.1 \%, \mathrm{P}=0.008)$ and higher in larynx cancers (28.1 vs $10.9 \%, \mathrm{P}=0.015)$ having significant difference, while the detection rate of HPV sequences was higher than that of viral protein in hypopharynx cancers
(40.0 vs $13.3 \%, P=0.107$ ) having no significant difference (Table I). Only a few cases (4/93) showed positive both in HPV sequences and proteins.

Correlations of HPV infections with tumor pathological and clinical grades. To address the possible correlation of HPV infection with tumor pathological classification, the prevalence of HPV was sub-grouped according to the tumor pathological grades. Based on the tumor cell differentiation pathologically, the enrolled cancers were divided into the groups of high, moderate and low differentiation. On the whole, the HPV positive rates were higher in the groups of high- $(46.6 \%, 13 / 28)$ and moderate-differentiation cancers $(46.0 \%, 23 / 51)$ than that in the group of low-differentiation cancers $(27.8 \%, 5 / 18)$ (Table II), but statistical analysis of $\chi^{2}$ did not reveal difference $(\mathrm{P}=0.513)$.

To see the correlation of HPV infection with the clinical progression, all enrolled cancers were classified according to their clinical grades. Generally, the HPV-positive rates in the groups of the clinical phase I, II, III and IV were $51.9(14 / 27)$, $37.1(13 / 35), 46.2(12 / 26)$ and $40.0 \%(2 / 5)$, respectively (Table III). Although the HPV-positive rate in the group of phase I was higher than those of the rest, statistic analysis of $\chi^{2}$ did not reveal significant difference. Furthermore, the HPV infections in the patients with or without metastasis were analyzed. The HPV-positive rates in the patients with metastasis $(53.3 \%, 8 / 15)$ and without metastasis $(42.3 \%$, $33 / 78$ ) were comparable, without statistic difference. It seems that HPV infection in the tumor tissues does not obviously affect the clinical progressions of the oral and throat carcinomas. 
Table III. Prevalence of HPV infection in the squamous head and neck carcinomas according to their clinical grades.

\begin{tabular}{lccccc}
\hline Clinic & Phase I (\%) & Phase II (\%) & Phase III $(\%)$ & Phase IV (\%) & P-value \\
\hline Larynx & $7 / 20(35.0)$ & $6 / 22(24.0)$ & $10 / 19(47.6)$ & $0 / 3(0.0)$ & 0.194 \\
Hypopharynx & $1 / 1(100.0)$ & $3 / 7(42.9)$ & $2 / 6(33.3)$ & $1 / 1(100.0)$ & 0.431 \\
Oropharynx & $2 / 2(100.0)$ & $0 / 1(0.0)$ & $0 / 1 \quad(0.0)$ & $1 / 1(100.0)$ & 0.172 \\
Lip & $4 / 4(100.0)$ & $4 / 5(80.0)$ & $0 / 0(0.0)$ & $0 / 0(0.0)$ & 1.0 \\
Total & $14 / 27(51.9)$ & $13 / 35(37.1)$ & $12 / 26(46.2)$ & $2 / 5(40.0)$ & 0.7 \\
\hline
\end{tabular}

Table IV. Prevalence of HPV infection in the squamous head and neck carcinomas based on the age groups.

\begin{tabular}{lcccccccc}
\hline Age (years) & $<30(\%)$ & $30-40(\%)$ & $40-50(\%)$ & $50-60(\%)$ & $60-70(\%)$ & $>70(\%)$ & P-value \\
\hline Larynx & - & $1 / 1(100.0)$ & $2 / 11(18.2)$ & $9 / 23(39.1)$ & $7 / 22(31.8)$ & $4 / 7$ & $(57.1)$ & 0.296 \\
Hypopharynx & - & - & $0 / 1 \quad(0.0)$ & $4 / 8$ & $(50.0)$ & $2 / 3(66.7)$ & $1 / 3(33.3)$ & 0.658 \\
Oropharynx & - & - & $1 / 2(50.0)$ & $2 / 2(100.0)$ & $0 / 1(0.0)$ & - & 0.233 \\
Lip & $1 / 1(100.0)$ & $2 / 2(100.0)$ & - & $2 / 2(100.0)$ & $1 / 1(100.0)$ & $2 / 3(66.7)$ & 0.690 \\
Total & $1 / 1(100.0)$ & $3 / 3(100.0)$ & $3 / 14(21.4)$ & $17 / 35(48.6)$ & $10 / 27(37.0)$ & $7 / 13(53.8)$ & 0.097 \\
\hline
\end{tabular}

Correlations of HPV infection with the gender, age and histories of tobacco and alcohol use of the patients. Among the enrolled 84 male patients, $40.5 \%$ (34/84) cases were HPV-positive, while predominantly more female patients $(77.8 \%, 7 / 9)$ showed HPV-positive, revealing an obvious gender-tendency. Among 7 HPV-positive female patients, 4 were lip carcinoma, 2 were larynx carcinoma and 1 was hypopharynx carcinoma. The median age of HPV-positive patients was 58 years, which was slightly older than that of HPV-negative patients (56.5 years old). The HPV-positive data among the different age groups are summarized in Table IV. Most cases were in the age groups of 40-50, 50-60, 60-70 and $>70$ years and HPV-positive rates in those four groups were $21.4,48.6,37.0$ and $53.8 \%$, respectively. There were only four patients $<40$ years, but all were HPV-positive. Statistical analysis did not reveal difference in HPV-positive rates among the age groups of 40-50, 50-60, $60-70$ and $>70$ years. Furthermore, the HPV infections among the patients with or without history of tobacco and alcohol use were analyzed. HPV detection rates in the groups of tobacco and alcohol use were $41.9(34 / 81)$ and $39.6 \%$ (21/53), respectively, which were lower than that in the groups without the history of tobacco $(58.3 \%, 7 / 12)$ and alcohol $(50.0 \%, 20 / 40)$ use, but there is no significant difference between the HPV-positive in the groups using tobacco or alcohol and not using tobacco or alcohol $(\mathrm{P}=0.289$ and 0.321$)$.

\section{Discussion}

The head and neck cancers in human refer to the malignant lesions at special anatomic sites, which include the lip, oral cavity, nose and para-nasal sinuses, naso-pharynx, oropharynx, hypopharynx and larynx. Although numerous studies have confirmed the presence of HPV genome and oncoproteins in head and neck SCCs, establishment of a stable linkage between HPV and a subset of SCCs is still difficult because of the heterogeneity of SCCs, and the fact that only a fraction of cases are HPV-associated. In this study, we provide data of HPV involvement in Chinese patients with SCCs at the sites of lip, oropharynx, and larynx. Overall 44.1\% (41/93) tested cases had HPV. These data are generally coincidental with the results of some previous studies of Chinese investigators (7), as well as that of international groups (8). In line with the previous observation (9), higher HPV detecting rates are observed in the patients who do not use tobacco or alcohol.

In our study, the HPV detection rates show a tumor siterelated distribution, which decrease from outside (lip) to inside (larynx). Although the enrolled case numbers of lip, oropharynx and hypopharynx cancers in this study are limited compared with that of larynx cancers, we still believe that this site-related HPV distribution in head and neck SCCs is meaningful, possibly reflecting the different exposure opportunity to HPVs. The exact transmission of HPV in oral mucosa remains uncertain, but direct skin to skin contact is believed to be essential, primarily by means of vaginal, anal and oral sex and less commonly by vertical transmission (10). Numerous studies have elucidated that oral HPV infection is closely linked with open mouthed kissing and oral sex (11-13). A case control study of 100 newly diagnosed patients with OSCC and 200 control patients without cancer has illustrated that a high lifetime number of vaginal-sex partners (26 or more) and a high lifetime number of oral-sex partners (6 or more) were associated with oropharyngeal cancer (14). Those comprehensive studies demonstrate the importance of oral sex in the HPV infection in head and neck SCCs. Although the oral sex histories in our Chinese patients are difficult to be addressed, the anatomic site-dependent HPV infections in the head and neck SCCs may reflect the importance of the direct contact.

Our data suggest that the HPV infection seems not to relate with either the pathological or clinical grades of head 
and neck SCCs. However, higher HPV detection rates are observed in patients with metastasis. Some earlier studies have reported that HPV-positive patients tended to present larger tumors and at a higher stage, while other have proposed opposite observation (15). Recent reports suggest that HPV-positive oropharyngeal cancers typically are detected at later stages (involvement of regional lymph nodes and distant metastasis) than HPV-negative cancers $(16,17)$. Patients with HPV-positive oropharyngeal cancers have consistently higher survival rates and better response to radiation therapy and chemotherapy and are less likely to experience progression and recurrence of tumors (18-21). In a meta-analysis of 37 studies, patients diagnosed with HPV-HNSCC had a lower risk of mortality and of recurrence as compared with patients with HPV-negative tumors (22). Whether HPV infection can be used as a prognostic predictor in Chinese head and neck SCCs needs further studies.

HPV16 is the most prevalent genotype in cervical carcinoma, and is also the most frequently detected HPV type in head and neck SCCs, found in up to $90 \%$ of HPV-positive cases $(2,23)$. Using the HPV Luminex technique that covers 26 HPV genotypes and HPV16- and 18-specific PCR, HPV16 and 18 DNAs were detected in 7 and 8 patients with head and neck SCC in this study. A previous study also showed that 7 (23.3\%) and $10(33.3 \%)$ of 30 Japanese and $11(36.7 \%)$ and $5(16.7 \%)$ of 30 Chinese samples contained HPV16 and HPV18, respectively (24). It highlights that HPV18 is another prevalent genotype in head and neck SCC in Northeast Asia, besides HPV16. Because of limited case numbers, HPV18 prevalence in head and neck SCC in the region and its clinical implication need to be investigated. Using HPV16/18 oncoprotein specific-IHC, more HPV-positive cases have been identified with the formalin-fixed and paraffinembedded tissues from head and neck SCC cases. Except for the possibility of relatively higher sensitivity of IHC assay, HPV DNA is probably easier to be detected in the fresh or fresh-frozen specimen (5). Our study also reveals a low overlapping ratio of coexistence of both HPV DNA and oncoprotein in the samples, in which only 4 out of 41 HPV-positive samples show doublepositive. Mismatch between persistence of viral genome and its encoding protein is common event in many persistent infectious viruses, especially in the integrated viruses with truncated viral genome fragments and with multiple integrating sites in host chromosome, such as high-risk HPVs $(25,26)$. Hence, the detection difference between HPV DNA and oncoproteins may not only represent the diversity of the testing sensitivities, but also reflect the complicated carcinogenesis of HPVs. Our data show an obviously predominant HPV16/18 oncoprotein positivity in lip cancers compared with SCC in other sites. Whether this phenomenon is related with relatively low HPV DNA detection in lip cancers in many other reports $(27,28)$ need further study. Although determination of p16 expression status with IHC has served as a reasonable surrogate marker for biologically relevant high-risk HPV infection (29), direct detection of high-risk E6/E7 mRNA or protein would be the ideal test for classifying a tumor as truly HPV-associated.

\section{Acknowledgements}

This study was supported by China Mega-Project for Infectious Disease (2011ZX10004-101 and 2008ZX10004-008) and the
SKLID Development Grant (2008SKLID102, 2011SKLID211 and 2012SKLID302). The Project Sponsored by the Young Scholar Scientific Research Foundation of China CDC (2012A102).

\section{References}

1. Gao N, Li Y, Li LJ and Wen YM: Clinical analysis of head and neck cancer cases in south-west China 1953-2002. J Int Med Res 37: 189-197, 2009

2. Marur S, D'Souza G, Westra WH and Forastiere AA: HPV-associated head and neck cancer: a virus-related cancer epidemic. Lancet Oncol 11: 781-789, 2010.

3. Machado J, Reis PP, Zhang T, et al: Low prevalence of human papillomavirus in oral cavity carcinomas. Head Neck Oncol 2: $6,2010$.

4. Hocking JS, Stein A, Conway EL, Regan D, Grulich A, Law M and Brotherton JML: Head and neck cancer in Australia between 1982 and 2005 show increasing incidence of potentially HPV-associated oropharyngeal cancers. Br J Cancer 104: 886-891, 2011.

5. Ramqvist T and Dalianis T: Oropharyngeal cancer epidemic and human papillomavirus. Emerg Infect Dis 16: 1671-1677, 2010.

6. Liu B, Lu Z, Wang P, Basang Z and Rao X: Prevalence of high-risk human papillomavirus types (HPV-16, HPV-18) and their physical status in primary laryngeal squamous cell carcinoma. Neoplasma 57: 594-600, 2010.

7. Sun P, Chen XP, Pei F, et al: Relationship between nasal inverted papilloma and human papillomavirus subtypes. Zhonghua Er Bi Yan Hou Tou Jing Wai Ke Za Zhi 45: 310-313, 2010.

8. Syrjänen S: Human papillomavirus (HPV) in head and neck cancer. J Clin Virol 32: 59-66, 2005.

9. Cleveland JL, Junger ML, Saraiya M, Markowitz LE, Dunne EF and Epstein JB: The connection between human papillomavirus and oropharyngeal squamous cell carcinomas in the United States: implications for dentistry. J Am Dent Assoc 142: 915-924, 2011.

10. Heck JE, Berthiller J, Vaccarella S, et al: Sexual behaviours and the risk of head and neck cancers: a pooled analysis in the International Head and Neck Cancer Epidemiology (INHANCE) consortium. Int J Epidemiol 39: 166-181, 2010.

11. D'Souza G, Zhang HH, D'Souza WD, Meyer RR and Gillison ML: Moderate predictive value of demographic and behavioral characteristics for a diagnosis of HPV16-positive and HPV16-negative head and neck cancer. Oral Oncol 46: 100-104, 2010.

12. Kreimer AR: Oral sexual behaviors and the prevalence of oral human papillomavirus infection. J Infect Dis 199: 1253-1254, 2009.

13. Rintala M, Grénman S, Puranen M and Syrjänen S: Natural history of oral papillomavirus infections in spouses: a prospective Finnish HPV family study. J Clin Virol 35: 89-94, 2006.

14. D'Souza G, Kreimer AR, Viscidi R, et al: Case-control study of human papillomavirus and oropharyngeal cancer. N Engl J Med 356: 1944-1956, 2007.

15. Fouret P, Martin F, Flahault A and Saint-Guily JL: Human papillomavirus infection in themalignant and premalignant head and neck epithelium. Diagn Mol Pathol 4: 122-127, 1995.

16. Chaturvedi AK, Engels EA, Anderson WF and Gillison ML: Incidence trends for human papillomavirus-related and -unrelated oral squamous cell carcinomas in the United States. J Clin Oncol 26: 612-619, 2008.

17. Watson M, Saraiya M, Ahmed F, et al: Using population-based cancer registry data to assess the burden of human papillomavirusassociated cancers in the United States: overview of methods. Cancer 113: 2841-2854, 2008.

18. Dayyani F, Etzel CJ, Liu M, Ho CH, Lippman SM and Tsao AS: Meta-analysis of the impact of human papillomavirus (HPV) on cancer risk and overall survival in head and neck squamous cell carcinomas (HNSCC). Head Neck Oncol 2: 15, 2010.

19. Fakhry C, Westra WH, Li S, et al: Improved survival of patients with human papillomavirus-positive head and neck squamous cell carcinoma in a prospective clinical trial. J Natl Cancer Inst 100: 261-269, 2008.

20. Ang KK, Harris J, Wheeler R, et al: Human papillomavirus and survival of patients with oropharyngeal cancer. N Engl J Med 336: 24-35, 2010

21. Settle K, Posner MR, Schumaker LM, et al: Racial survival disparity in head and neck cancer results from low prevalence of human papillomavirus infection in black oropharyngeal cancer patients. Cancer Prev Res 2: 776-781, 2009. 
22. Ragin CC and Taioli E: Survival of squamous cell carcinoma of the head and neck in relation to human papillomavirus infection: review and meta analysis. Int J Cancer 121: 1813-1820, 2007.

23. Psyrri A and DiMaio D: Human papillomavirus in cervical and head-and-neck cancer. Nat Clin Pract Oncol 15: 24-31, 2008.

24. Tang X, Jia L, Ouyang J and Takagi M: Comparative study of HPV prevalence in Japanese and North-east Chinese oral carcinoma. J Oral Pathol Med 32: 393-398, 2003.

25. Pett $M$ and Coleman N: Integration of high-risk human papillomavirus: a key event in cervical carcinogenesis? J Pathol 212: 356-367, 2007.

26. Kraus I, Driesch C, Vinokurova S, et al: The majority of viralcellular fusion transcripts in cervical carcinomas cotranscribe cellular sequences of known or predicted genes. Cancer Res 68: 2514-2522, 2008.
27. Paz IB, Cook N, Odom-Maryon T, Xie Y and Wilczynski SP: Human papillomavirus (HPV) in head and neck cancer. An association of HPV 16 with squamous cell carcinoma of Waldeyer's tonsillar ring. Cancer 79: 595-604, 1997.

28. Shimizu M, Adachi A, Zheng S, et al: Detection of various types of human papillomavirus DNA, mainly belonging to the cutaneous-group, more frequently in normal tissue than in squamous cell carcinomas of the lip. J Dermatol Sci 36: 33-39, 2004.

29. Smeets SJ, Hesselink AT, Speel EJ, et al: A novel algorithm for reliable detection of human papillomavirus in paraffin embedded head and neck cancer specimen. Int J Cancer 121: 2465-2472, 2007. 